\title{
Estudio de la relación de los terceros molares superiores retenidos y el seno maxilar en radiografías panorámicas y tomografía (CBCT).
}

\section{Study of the relationship of retained upper third molars and maxillary sinus in panoramic radiographs and tomography (CBCT).}

\author{
Diego Vázquez,* Beatriz Subirán, ${ }^{\ddagger}$ Martin Pujol, ${ }^{\ddagger}$ Alejandra Antoniuk, ${ }^{\S}$ Leonard Nart, ${ }^{\S}$ \\ Lorena Benítez, ${ }^{\S}$ Walter Moyano, " Luis Rannelucci," Pedro Hecht ${ }^{\llbracket}$
}

\section{RESUMEN}

Objetivo: Evaluar la eficacia de la radiografía panorámica para establecer la relación de los terceros molares superiores y la cortical inferior del seno maxilar, en comparación con la tomografía computarizada de haz cónico (CBCT). Material y métodos: El trabajo incluyó 72 pacientes, se estudiaron 34 terceros molares superiores retenidos izquierdos y 38 derechos. Se realizaron mediciones con el programa para establecer la distancia entre la cortical inferior del seno maxilar y el ápice del tercer molar superior a partir de la imagen de la radiografía panorámica en lo que se estableció como parámetros: «a distancia, en contacto y dentro del piso de seno maxilar». Luego, con los cortes oblicuos o paraaxiales de la tomografía computarizada (CBCT) se observó la verdadera ubicación en el plano bucopalatino. Resultados: Del total de los terceros molares superiores 34 fueron izquierdas (47.22\%) y 38 derechas (52.78\%). En la Rx. Panorámica, 18 casos (25\%) se encontraron a distancia al seno maxilar, 12 en contacto (16.7\%) y 42 por dentro $(58.3 \%)$ del seno maxilar. En la tomografía se observaron 20 casos $(27.8 \%)$ a distancia del conducto, $16(22.2 \%)$ en contacto y $36(50 \%)$ por dentro del seno maxilar. Se observaron diferencias estadísticamente significativas $(\mathrm{p}<0.05)$ entre la Rx. panorámica y la tomografía $(\mathrm{CBCT})$ de los terceros molares que se hallan por dentro del seno maxilar. Conclusión: A partir de los resultados obtenidos, podemos establecer que la radiografía panorámica no permite conocer la verdadera relación entre la cortical inferior del seno maxilar y los terceros molares superiores.

Palabras clave: Radiografía panorámica, tomografía computarizada, haz cónico, TCAC, tercer molar superior, retención dentaria.

\section{ABSTRACT}

Objective: To evaluate the effectiveness of panoramic radiography to establish the relationship of the upper third molars and the inferior cortical of the maxillary sinus, in comparison with the Cone Beam Computed Tomography (CBCT). Material and methods: The work included 72 patients, 34 upper left maxillary molars and 38 rights were studied. Measurements were made with the program to establish the distance between the lower cortex of the maxillary sinus and the apex of the upper third molar from the image of the panoramic radiograph in what was established as parameters: «at a distance, in contact and inside the floor of maxillary sinus». Then with the oblique or paraaxial slices of the computed tomography (CBCT) the true location in the bucco-palatal plane was observed. Results: Of the total upper third molars, 34 were left (47.22\%) and 38 right (52.78\%). In the panoramic $X$-ray 18 cases (25\%) were found at distance to the maxillary sinus, 12 in contact (16.7\%) and 42 inside (58.3\%) of the maxillary sinus. The tomography showed 20 cases (27.8\%) at a distance from the canal, 16 (22.2\%) in contact and 36 $(50 \%)$ inside the maxillary sinus. Statistically significant differences were observed $(p<0.05)$ between the Rx. panoramic and tomography $(C B C T)$ of the third molars that are inside the maxillary sinus. Conclusion: From the results obtained, we can establish that the panoramic radiograph does not allow knowing the true relationship between the inferior cortical of the maxillary sinus and the upper third molars.

Keywords: Panoramic radiography, computed tomography, cone beam, CBCT, upper third molar, dental retention.

\footnotetext{
* Profesor Titular. Cátedra de Diagnóstico por Imágenes.

‡ Profesor Adjunto. Cátedra de Diagnóstico por Imágenes.

$\S$ Jefe de Trabajos Prácticos. Cátedra de Diagnóstico por Imágenes.

" Ayudante de primera. Cátedra de Diagnóstico por Imágenes.

^ Jefe de Trabajos Prácticos. Cátedra de Odontología Legal y Forense.

Facultad de Odontología de la Universidad de Buenos Aires, Argentina.

Recibido: 21 Octubre 2019.

Aceptado para publicación: 18 Noviembre 2019.
}

\section{INTRODUCCIÓN}

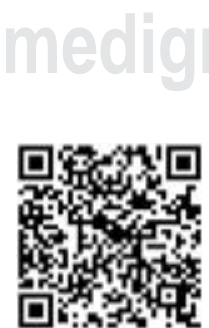

Se considera que un diente retenido es una anomalía denStaria de desarrollo, en la cual una vez que llega la etapa normal de erupción, las estructuras dentarias permanecen en el interior de los maxilares, sin alterar la integridad del saco pericoronario, pudiendo clasificarse en retención subgingival cuando está rodeado por mucosa gingival e intraósea rodeado por tejido óseo, siendo ésta de mayor profundidad. ${ }^{1}$ 
Se pudo evidenciar que durante el trascurso de los años, el hombre ha modificado sus hábitos alimenticios, provocando en el mismo una reducción en la función masticatoria debido a la disminución del tamaño de los músculos y huesos que cumplen dicha función, dando por resultado un aumento en los dientes retenidos por la falta de espacio en los maxilares una vez llegada la etapa cronológica normal de erupción. Las maloclusiones ocupan un lugar de prevalencia en lo que respecta a las anomalías dentarias de desarrollo y se establece que son provocadas por órganos dentarios retenidos.

Estadísticamente, se considera que los terceros molares superiores son las estructuras dentarias que se hallan con mayor frecuencia en retención, encontrándose con mayor incidencia en la segunda década de vida sin evidenciar diferencias entre hombres y mujeres según la bibliografía consultada. ${ }^{1,2}$

Cuando los gérmenes están en una mala posición, se les considera como un factor de retención, también la alteración de las fuerzas de erupción, la disminución del tamaño normal de la arcada dentaria, la esclerosis ósea, hiperplasias gingivales fibrosas, pérdida prematura de los dientes temporales, presencia de dientes supernumerarios, tumores, quistes, dilaceraciones y anquilosis también son consideradas como presuntas alteraciones que causarían esta anomalía.
Los terceros molares superiores retenidos se caracterizan por poder generar quistes cuando el saco pericoronario que recubre al diente se llena de fluidos, incrementando su tamaño hasta poder formar una estructura quística, alterando estructuras óseas y dentarias adyacentes. Se recomienda la extracción quirúrgica del tercer molar retenido a edades tempranas. ${ }^{2,3}$

La radiografía panorámica es una técnica sencilla de realizar y de gran utilidad para diagnosticar posibles patologías en los maxilares. Con la incorporación de la digitalización en radiología, se ha logrado mejorar la calidad y definición de las imágenes. ${ }^{4,5}$ La tomografía computarizada de haz cónico o CBCT (Cone Beam Computed Tomography), fue desarrollada para obtener imágenes de las estructuras de cabeza y cuello en tres dimensiones con una dosis de radiación menor que la tomografía computarizada multicortes. Este tipo de tomografía emite radiación en forma de haz cónico adquiriendo la imagen un volumen 3D. ${ }^{4,6}$

El objetivo del trabajo es evaluar la eficacia de la radiografía panorámica para establecer la relación de los terceros molares superiores y la cortical inferior del seno maxilar, en comparación con la tomografía computada (CBCT) en la ciudad de Buenos Aires en Argentina.

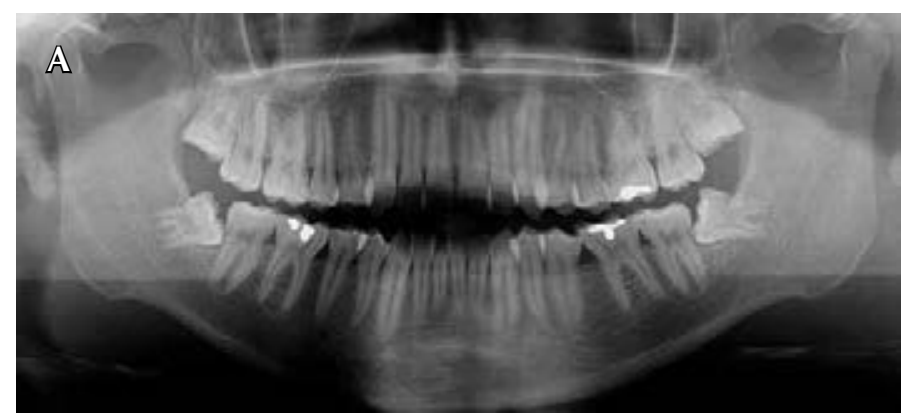

Figura 1:

A) Radiografía panorámica. Se observa el órgano 1.8 en contacto con la cortical del seno maxilar. B) CBCT corte panorexis. Se observa el órgano

1.8 a distancia de la cortical

inferior del seno maxilar.

Se observa en el corte curvo axial. C) CBCT corte oblicuo.

Se observa distancia entre la cortical del seno y el órgano 1.8.
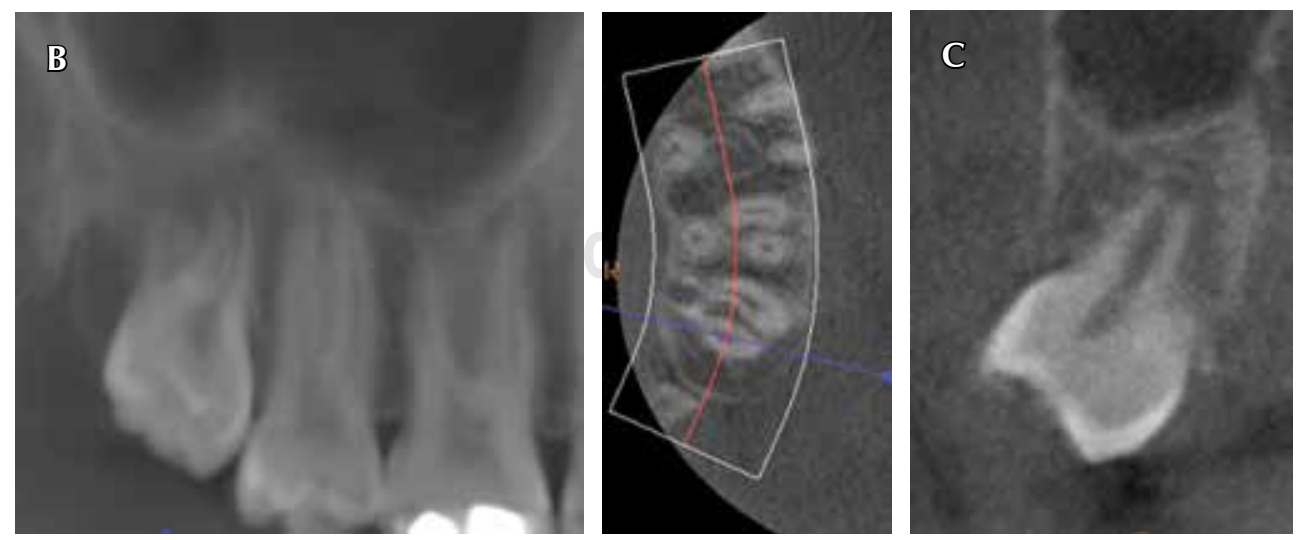

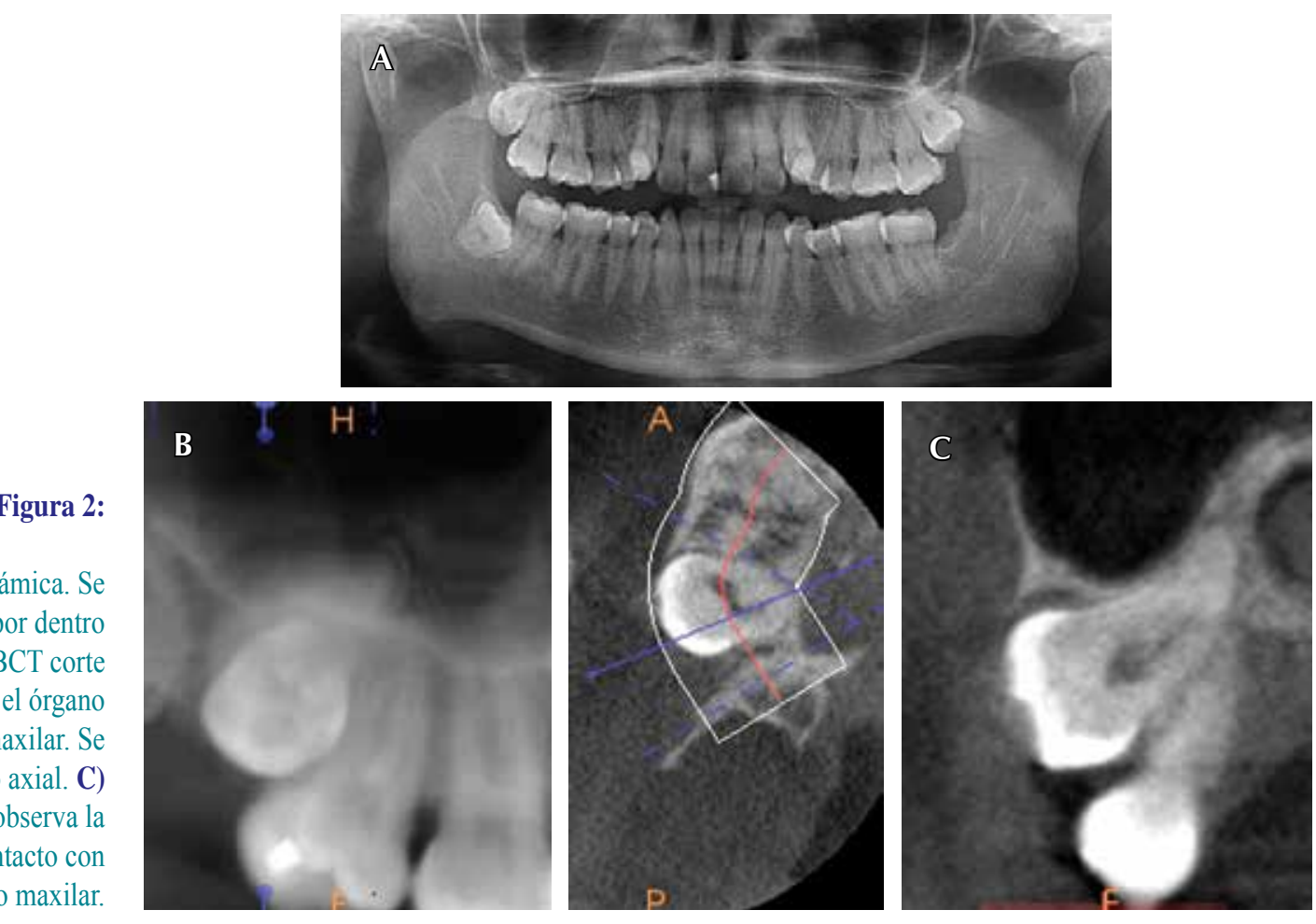

A) Radiografía panorámica. Se observa el órgano 1.8 por dentro del seno maxilar. B) CBCT corte panorexis. Se observa el órgano 1.8 por dentro del seno maxilar. Se observa en el corte curvo axial. C) CBCT corte oblicuo. Se observa la raíz del órgano 1.8 en contacto con

la cortical del seno maxilar.

\section{MATERIAL Y MÉTODOS}

Se realizó un estudio retrospectivo descriptivo en el que se observaron los registros de las imágenes digitales de una muestra de 72 radiografías y tomografías seleccionados al azar, siendo derivados para la toma de imágenes por odontólogos para la exodoncia de los terceros molares superiores.

Criterios de inclusión: 18 años hasta los 50 años, de sexo masculino y femenino que puedan quedarse inmóvil durante la toma de los estudios imagenológicos. Criterios de exclusión: mujeres embarazadas.

Las adquisiciones de las tomografías (CBCT) y radiografías panorámicas se realizaron en un equipo marca Carestream 9000c 3D de origen Francés, con un tamaño de vóxel de $0.7 \times 0.7 \times 7 \mathrm{~mm}^{3}$ y un tamaño de Fov de $50 \times 37 \mathrm{~mm}$. Se utilizó para realizar las radiografías panorámicas, valores que fueron desde los 63 a $88 \mathrm{kv}$, de 8 a $10 \mathrm{~mA}$, y 10 a 15 segundos de tiempo de exposición por cada radiografía según contextura, tamaño y edad del paciente. Para la realización de las tomografías CBCT se utilizaron valores desde los $63 \mathrm{Kv}$ hasta los 90 kv, de 8 hasta 10 mA, y 11 a 18 segundos de tiempo de exposición por estudio según la contextura, tamaño y edad de cada paciente.
Se utilizó el programa del equipo Carestream 9000c $3 \mathrm{D}$ en el que se editaron en los cortes paraxiales u oblicuos de la imagen en los maxilares superiores en la zona correspondiente a los terceros molares.

En el trabajo se observaron 72 pacientes, en los cuales se estudiaron 34 terceros molares superiores retenidos izquierdos y 38 molares superiores retenidos derechos. Se realizaron mediciones con el software para establecer la distancia entre la cortical inferior del seno maxilar y el ápice del tercer molar superior a partir de la imagen de la radiografía panorámica en lo que se estableció: «a distancia, en contacto y dentro» como parámetros. Luego, con los cortes oblicuos o paraaxiales de la tomografía computada (CBCT), se observó la verdadera ubicación en el plano buco-palatino con la herramienta del software para medir la ubicación precisa de los terceros molares superiores retenidos por personal calibrado en la sala de informes de la Cátedra de Diagnóstico por Imágenes de la Facultad de Odontología de la Universidad de Buenos Aires (Figuras 1 y 2).

\section{RESULTADOS}

En el estudio se pudo observar que se hallaron 34 molares superiores izquierdos (47.22\%) y 38 molares superiores 


\begin{tabular}{ccccccccc} 
& \multicolumn{2}{c}{ Tercer molar } & & \multicolumn{2}{c}{ Panorámica } & & \multicolumn{2}{c}{ CBCT } \\
\cline { 2 - 3 } Lado & A distancia & En contacto & & Dentro & A distancia & & En contacto & Dentro \\
\hline Total & 18 & 12 & & 42 & 20 & & 16 & 36
\end{tabular}

derechos (52.78\%). En las radiografías panorámicas se hallaron 18 casos (25\%) con distancia al seno maxilar, 12 en contacto con el seno maxilar (16.7\%) y 42 por dentro del seno maxilar (58.3\%).

En la tomografía CBCT se observaron 20 casos (27.8\%) a distancia del conducto, 16 (22.2\%) en contacto y 36 (50\%) por dentro del seno maxilar.

El análisis estadístico incluyó comparaciones apareadas no paramétricas (test de pares apareados de Wilcoxon) para las variables de relación entre el tercer molar superior y la cortical del seno maxilar en la radiografía panorámica o CBCT. Se observaron diferencias altamente significativas al nivel $p<0.05$ entre los terceros molares superiores derechos e izquierdos, que se hallan por dentro del seno maxilar. En las comparaciones apareadas de los terceros molares superiores entre las radiografías panorámicas y CBCT a distancia y en contacto no se evi-

\section{Comparación CBCT vs panorámica}

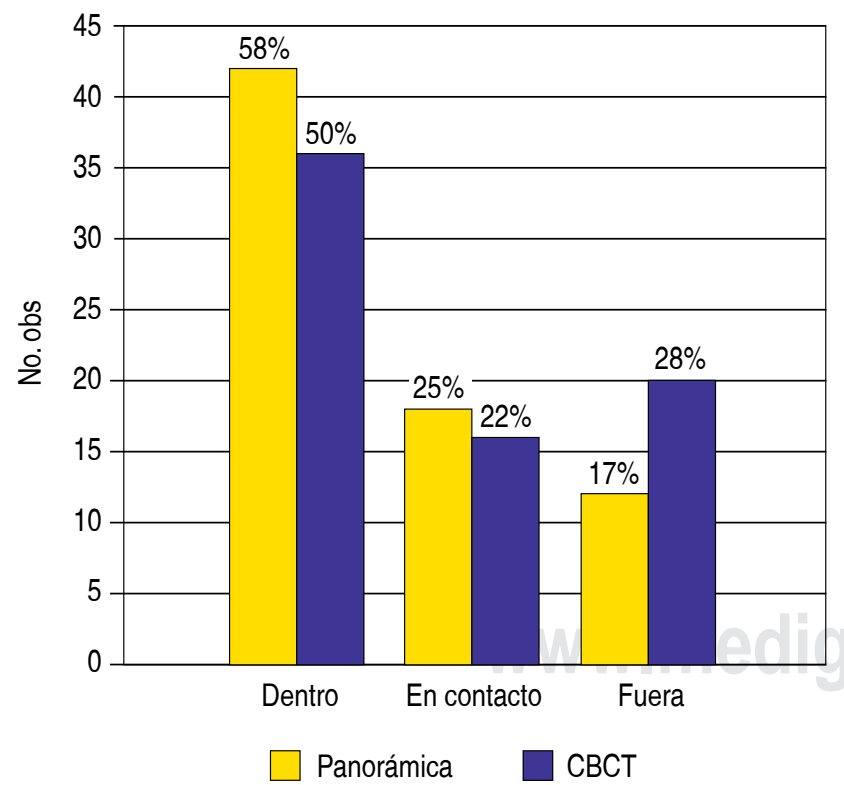

Figura 3: Relación de los terceros molares superiores con respecto a la cortical del seno maxilar evaluada en radiografías panorámicas y CBCT. denciaron diferencias estadísticamente significativas. No se hallaron diferencias significativas según el lado (derecho o izquierdo) y según el sexo (Figura 3).

\section{DISCUSIÓN}

A partir de la incorporación de la tomografía computarizada de haz cónico (CBCT) en odontología, se puede obtener exactitud para diagnosticar posibles patologías y la planificación de los tratamientos a partir de la alta resolución y calidad de imágenes que ofrece este tipo de equipamiento. Nuestro estudio nos permitió trabajar con mediciones uno a uno en la relación existente entre las raíces de los terceros molares superiores y la cortical inferior del seno maxilar, pudiendo observar en los cortes oblicuos estructuras de diferentes ubicaciones con respecto a la bidimensionalidad que nos ofrece la radiografía panorámica. ${ }^{7,8}$

Según la bibliografía consultada, se han realizado numerosos estudios en lo que respecta a la relación de los terceros molares inferiores y su relación con el conducto dentario inferior, pero no se evidencia el estudio de los terceros molares superiores con respecto al seno maxilar. ${ }^{2,9}$

\section{CONCLUSIONES}

A partir de los resultados obtenidos, podemos establecer que la radiografía panorámica no permite conocer la verdadera relación entre la cortical inferior del seno maxilar y los terceros molares superiores y que la tomografía CBCT utilizada en odontología nos permite dar exactitud en el diagnóstico de la relación de la ubicación dentaria y las estructuras anatómicas asociadas.

\section{BIBLIOGRAFÍA}

1. Vázquez D, Martínez M, Hecht P. Frecuencia de las retenciones dentarias en radiografías panorámicas. Presentación de un estudio en 100 pacientes. Acta Odontol Venez. 2013; 51 (1).

2. Vázquez D, Subiran B, Osende N, Estévez A, Vautier M, Hecht P. Estudio comparativo de la relación de los terceros molares inferiores retenidos con el conducto dentario inferior en radiografías panorámicas y tomografías Cone Beam. Revista Científica Odontológica. 2016; 12 (1): 14-18. 
3. De Coster PJ, Mortier G, Marks LA, Martens LC. Cranial suture biology and dental development: genetic and clinical perspectives. J Oral Pathol Med. 2007; 36 (8): 447-455.

4. Araki K, Maki K, Seki K, Sakamaki K, Harata Y, Sakaino R et al. Characteristics of a newly developed dentomaxillofacial X-ray cone beam CT scanner. System configuration and physical properties. Dentomaxillofac Radiol. 2004; 33: 51-59.

5. Kawada S, Koyasu K, Zholnerovskaya El, Oda S. Analysis of dental anomalies in the Siberian mole, Talpa altaica (Insectivora, Talpidae). Arch Oral Biol. 2006; 51 (11): 1029-1039.

6. Quereshy FA, Savell TA, Palomo JM. Applications of cone beam computed tomography in the practice of oral and maxillofacial surgery. J Oral Maxillofac Surg. 2008; 66: 791-796.

7. Vázquez D, Bruno I, Ramírez MJ, Martínez B, Carbajal E, Martínez M. Estudio de prevalencia de las patologías dentarias de desarrollo en radiografías panorámicas. Rev Fac Odont UBA. 2008; 23 (54/55): 9-12.

8. Ezoddini AF, Sheikhha MH, Ahmadi H. Prevalence of dental developmental. anomalies: a radiographic study. Community Dent Health. 2007; 24 (3): 140-144.

9. Shahbazian M, Vandewoude C, Wyatt J, Jacobs R. Comparative assessment of panoramic radiography and $\mathrm{CBCT}$ imaging for radio diagnostics in the posterior maxilla. Clin Oral Investig. 2014; 18 (1): 293-300.

\section{Correspondencia:}

Diego Jorge Vázquez

E-mail:dr.diegovazquez@hotmail.com 